\title{
Iterated Logarithm Laws on GLM Randomly Censored with Random Regressors and Incomplete Information ${ }^{\#}$
}

\author{
Qiang Zhu ${ }^{1}$, Zhihong Xiao ${ }^{1}$, Guanglian Qin ${ }^{1}$, Fang Ying ${ }^{2}$ \\ ${ }^{1}$ Statistics Research Institute, Huazhong Agricultural University, Wuhan, China \\ ${ }^{2}$ School of Science, Hubei University of Technology, Wuhan, China \\ E-mail: xzhhsfxym@mail.hzau.edu.cn \\ Received December 8, 2010; revised January 26, 2011; accepted January 29, 2011
}

\begin{abstract}
In this paper, we define the generalized linear models (GLM) based on the observed data with incomplete information and random censorship under the case that the regressors are stochastic. Under the given conditions, we obtain a law of iterated logarithm and a Chung type law of iterated logarithm for the maximum likelihood estimator (MLE) $\hat{\beta}_{n}$ in the present model.
\end{abstract}

Keywords: Generalized Linear Model, Incomplete Information, Stochastic Regressor, Iterated Logarithm Laws

\section{Introduction}

The generalized linear model(GLM) was put forward by Nelder and Wedderburn [1] in 1970s and has been studied widely since then. The maximum likelihood estimator (MLE) $\hat{\beta}_{n}$ of the parameter vector $\beta$ in GLM was given and its strong consistency and asymptotic normality were discussed by Fahrmeir and Kaufmann [2] in 1985.The randomly censored model with the incomplete information was presented by Elperin and Gertsbakin [3] in 1988.The analysis of the randomly censored data with incomplete information has become a new branch of the Mathematical Statistics. Xiao and Liu [4] in 2008 discussed the strong consistency and the asymptotic normality of MLE $\hat{\beta}_{n}$ of GLM based on the data with random censorship and incomplete information. Xiao and Liu [5] discussed laws of iterated logarithm for quasi-maximum likelihood estimator of GLM in 2008, meanwhile, Xiao and Liu [6] in 2009 discussed laws of iterated logarithm for maximum likelihood estimator of generalized linear model randomly censored with incomplete information under the regressors given. However, Lai and Wei [8], Zeger and Karim [9] have studied the linear regression model under the case that the regressors are stochastic. In the practical application, espe-

*Corresponding Author.

\#Foundation items: supported by Huazhong Agricultural University Doctoral Fund (52204-02067) and Interdisciplinary Fund (2008 xkjc008 and Torch Plan Fund (2009XH003). cially in the biomedical social sciences, the regressors in GLM are often stochastic, Fahrmeir [10] investigated GLM with the regressors $X_{1}, \cdots, X_{n}$ which are independent and identically distributed and gave MLE of matrix parameter without proof under the given conditions. Ding and Chen [11] in 2006 gave asymptotic properties of MLE in GLM with stochastic regressors. So, in the present paper, we will investigate the law of iterated logarithm and the Chung type law of iterated logarithm for maximum likelihood estimator of generalized linear model randomly censored with incomplete information under the case that regressive variables $X_{i}, i \geq 1$ are independent but not necessarily identically distributed.

From a statistical perspective, the importance of those laws stem from the fact that the first one gives in an asymptotic sense the smallest $100 \%$ confidence interval for the parameter, while the second one gives an almost sure lower bound on the accuracy that the estimator can achieve.

\section{Model with the Random Regressor}

Suppose that the respondence variables $Y_{i}, i=1,2, \cdots, n$ are one dimension random variables, and regressor variable $X_{i}, i=1,2, \cdots, n$ are q-dimension random variables which have the distribution functions $K_{i}, i=1,2, \cdots$, respectively. Here, $x_{i}$ is the observation value of $X_{i}, X_{i} \in$ $\aleph_{i}$. Write $\aleph=\bigcup_{i=1}^{\infty} \aleph_{i}$. Suppose that the observations $\left(Y_{i}, X_{i}\right), i=1,2, \cdots$, are mutually independent and satisfy. 
1) The regression equation:

$$
E\left[Y_{i} \mid X_{i}=x_{i}\right]=m\left(x_{i}^{T} \beta\right), i \geq 1
$$

where the unknown parameter $\beta \in B \subset \mathbb{R}^{q}$.

2) The conditional distribution of $Y_{i}$ under $X_{i}=x_{i}$ is the exponent distribution, i.e.

$$
P\left(Y_{i} \in d y \mid X_{i}=x_{i}\right)=C(y) \exp \left\{\theta_{i} y-b\left(\theta_{i}\right)\right\} \mu(d y), i \geq 1
$$

where $\mu$ is a $\sigma$-finite measure, parameter $\theta_{i} \in \Theta$,

$(i=1,2, \cdots, n), \Theta \equiv\left\{\theta: 0<\int C(y) \exp \{\theta y\} \mu(d y)<\infty\right\}$ is the natural parameter space and $\Theta_{0}$ is the interior of $\Theta$. Since this conditional density integrates to 1 , we see that $b\left(\theta_{i}\right)=\int C(\pi) \exp \left\{\theta_{i} y\right\} \mu(d y)$, from which the standard expressions for the conditional mean $E\left[Y_{i} \mid X_{i}=x_{i}\right]=\dot{b}\left(\theta_{i}\right)$, and the variance, $\operatorname{Var}\left[Y_{i} \mid X_{i}=x_{i}\right]$ $=\ddot{b}\left(\theta_{i}\right)$, where $\dot{b}(\bullet), \ddot{b}(\bullet)$ denote the first and second derivatives of $b(\bullet)$, respectively.

Suppose that the censor random variables $U_{i}, i=$ $1,2, \cdots, n$ are mutually independent but not necessarily identically distributed, with the distribution function $G_{i}(u)$ and $d G_{i}(u)=g_{i}(u) \mu(d u)$. Denote $K_{i}(d x)=$ $\tau_{i}(x) \mu(d x), i=1,2, \cdots, n$. Suppose that $U_{i}$ is independent of $\left(Y_{i}, X_{i}\right)$

For $i=1,2, \cdots, n$, let $\alpha_{i}=I_{\left\{Y_{i}<U_{i}\right\}}$,

$\delta_{i}=\left\{\begin{array}{l}0, \text { if } Y_{i}<U_{i}, \text { but the real value of } Y_{i} \text { isn't observed }, \\ 1, \quad \text { else, }\end{array}\right.$

$$
Z_{i}=\left\{\begin{array}{l}
Y_{i}, \text { if } \alpha_{i}=1, \delta_{i}=1 \\
U_{i}, \quad \text { otherwise }
\end{array}\right.
$$

Obviously, $\left\{\left(Z_{i}, \alpha_{i}, \delta_{i}, X_{i}\right), i=1,2, \cdots\right\}$ is a mutually independent and observable sample. The conditional density and distribution function of $Y_{\mathrm{i}}$ under $X_{i}=x_{i}$ are respectively denoted as

$$
\begin{gathered}
f\left(y ; x_{i}^{T} \beta\right) \equiv C(y) \exp \left\{x_{i}^{T} \beta y-b\left(x_{i}^{T} \beta\right)\right\} \\
F\left(z ; x_{i}^{T} \beta\right) \equiv \int_{-\infty}^{z} C(y) \exp \left\{x_{i}^{T} \beta y-b\left(x_{i}^{T} \beta\right)\right\} d \mu(y) \\
=P\left(Y_{i}<z \mid X_{i}=x_{i}\right)
\end{gathered}
$$

Let $\bar{G}_{i}(z)=1-G_{i}(z)$,

$$
\bar{F}\left(z ; x_{i}^{T} \beta\right)=1-F\left(z ; x_{i}^{T} \beta\right), \quad i=1,2, \cdots, n .
$$

Suppose

$$
\begin{aligned}
& P\left(\delta_{i}=1 \mid Y_{i}=y, U_{i}=u, X_{i}=x\right)=p, \\
& \text { if } y<u, \forall x \in \aleph_{i}, \\
& P\left(\delta_{i}=0 \mid Y_{i}=y, U_{i}=u, X_{i}=x\right)=1-p, \\
& \text { if } y<u, \forall x \in \aleph_{i},
\end{aligned}
$$

where $0 \leq p \leq 1$. This assumption came from T. Elperin and I. Gertsbak, [3]. In the reliability study, the instant of an item's failure is observed if it occurs before a randomly chosen inspection time and the failure is signaled. Otherwise, the experiment is terminated at the instant of inspection during which the true state of the item is discovered. T. Elperin and I. Gertsbak, assumed that the failure time of every item was signaled randomly with probability $p$ before the randomly chosen inspection time. Then, we have

$$
\begin{aligned}
& P\left(Y_{i}<y, U_{i}<u \mid X_{i}=x\right) \\
& =P\left\{Y_{i}<y \mid X_{i}=x\right\} P\left\{U_{i}<u\right\}, \forall y, u, x
\end{aligned}
$$

Without loss of generality, assuming that $X_{i}$ is discrete, we have

$$
P\left(Y_{i}<y, U_{i}<u \mid X_{i}=x\right)=P\left\{Y_{i}<y \mid X_{i}=x\right\} P\left\{U_{i}<u\right\}
$$

We first give the following propositions.

Proposition 2.1. Under the regular assumptions above, we have

$$
\begin{gathered}
P\left(Z_{i}<z, \alpha_{i}=1, \delta_{i}=1 \mid X_{i}=x\right) \\
=p \int_{-\infty}^{z} \bar{G}_{i}(y) f_{i}(y) \mu(d y), \\
P\left(Z_{i}<z, \alpha_{i}=1, \delta_{i}=0 \mid X_{i}=x\right) \\
=(1-p) \int_{-\infty}^{z} F_{i}\left(y ; x_{i}^{T} \beta\right) d G_{i}(y), \\
P\left(Z_{i}<z, \alpha_{i}=0 \mid X_{i}=x\right) \\
=\int_{-\infty}^{z} \bar{F}_{i}\left(y ; x_{i}^{T} \beta\right) d G_{i}(y) .
\end{gathered}
$$

Proof. We only show (2.6) for the discrete case, the continuous case can be shown in the way similar to that of the discrete case.

$$
\begin{aligned}
& P\left(Z_{i}<z, \alpha_{i}=1, \delta_{i}=1 \mid X_{i}=x\right)=E\left[I_{\left\{Y_{i}<z\right\}} I_{\left\{Y_{i}<U_{i}\right\}} E\left[I_{\left\{\delta_{i}=1\right\}} \mid Y_{i}, U_{i}, X_{i}=x_{i}\right] \mid X_{i}=x_{i}\right] \\
& =\int_{-\infty}^{z} \int_{y}^{\infty} P\left(\delta_{i}=1 \mid Y_{i}=y, U_{i}=u, X_{i}=x_{i}\right) P\left(Y_{i} \in d y, U_{i} \in d u \mid X_{i}=x_{i}\right) \\
& =p \int_{-\infty}^{z} \bar{G}_{i}(y) d F_{i}(y)=p \int_{-\infty}^{z} \bar{G}_{i}(y) f_{i}(y) \mu(d y)
\end{aligned}
$$


where (2.9) follows from (2.3) and (2.5). Similarly, we can demonstrate (2.7) and (2.8).

Suppose that $z_{i}$ is the observation of $Z_{i}, \bar{\alpha}_{i}$ is the observation of $\alpha_{i}, \bar{\delta}_{i}$ is the observation of $\delta_{i},(2.6)$, (2.7) and (2.8) imply that for all $i \geq 1$, the conditional distribution of $\left(Z_{i}, \alpha_{i}, \delta_{i}\right)$ under $X_{i}=x_{i}$ is the following

$\left\{\left[p \bar{G}_{i}\left(z_{i}\right) f\left(z_{i} ; x_{i}^{T} \beta\right)\right]^{\bar{a}_{i} \bar{\delta}_{i}}\left[(1-p) F\left(z_{i} ; x_{i}^{T} \beta\right) g_{i}\left(z_{i}\right)\right]^{\bar{\alpha}_{i}\left(1-\bar{\delta}_{i}\right)}\right.$.

$\left.\left[\bar{F}\left(z_{i} ; x_{i}^{T} \beta\right) g_{i}\left(z_{i}\right)\right]^{\left(1-\bar{\alpha}_{i}\right)} \mu\left(d z_{i}\right)\right\}$

Let

$Z_{(n)}=\left(Z_{1}, \cdots, Z_{n}\right), z_{(n)}=\left(z_{1}, \cdots, z_{n}\right), \alpha_{(n)}=\left(\alpha_{1}, \cdots, \alpha_{n}\right)$,

$\bar{\alpha}_{(n)}=\left(\bar{\alpha}_{1}, \cdots, \bar{\alpha}_{n}\right), \delta_{(n)}=\left(\delta_{1}, \cdots, \delta_{n}\right), \bar{\delta}_{(n)}=\left(\bar{\delta}_{1}, \cdots, \bar{\delta}_{n}\right)$,

$X_{(\infty)}=\left(X_{1}, X_{2}, \cdots\right), X_{(n)}=\left(X_{1}, \cdots, X_{n}\right), x_{(n)}=\left(x_{1}, \cdots, x_{n}\right)$,

$x_{(\infty)}=\left(x_{1}, x_{2}, \cdots\right)$.

We easily get the following proposition.
Proposition 2.2. For all $n \geq 1$, we have

$$
\begin{aligned}
& P\left(Z_{(n)}<Z_{(n)}, \alpha_{(n)}=\bar{\alpha}_{(n)}, \delta_{(n)}=\bar{\delta}_{(n)} \mid X_{\infty}=x_{\infty}\right) \\
& =P\left(Z_{(n)}<Z_{(n)}, \alpha_{(n)}=\bar{\alpha}_{(n)}, \delta_{(n)}=\bar{\delta}_{(n)} \mid X_{(n)}=x_{(n)}\right) \\
& =\prod_{i=1}^{n} P\left(Z_{i}<z_{i}, \alpha_{i}=\bar{\alpha}_{i}, \delta_{i}=\bar{\delta}_{i} \mid X_{i}=x_{i}\right)
\end{aligned}
$$

and

$$
\begin{aligned}
& P\left(Z_{i}<z_{i}, \alpha_{i}=\bar{\alpha}_{i}, \delta_{i}=\bar{\delta}_{i} \mid X_{\infty}=x_{\infty}\right) \\
& =P\left(Z_{i}<z_{i}, \alpha_{i}=\bar{\alpha}_{i}, \delta_{i}=\bar{\delta}_{i} \mid X_{(n)}=x_{(n)}\right) \\
& =P\left(Z_{i}<z_{i}, \alpha_{i}=\bar{\alpha}_{i}, \delta_{i}=\bar{\delta}_{i} \mid X_{(i)}=x_{(i)}\right), \forall i \geq 1,
\end{aligned}
$$

where $Z_{(n)}<Z_{(n)}$ means $Z_{i}<Z_{i}$ for $1 \leq i \leq n$.

Remark 2.1. Proposition 2.2 implies that under $P\left(\bullet X_{\infty}=X_{\infty}\right), U_{i}, i \geq 1$ are mutually independent and so are $Y_{i}, i \geq 1$, and $\left(Z_{i}, \alpha_{i}, \delta_{i}\right), i \geq 1$.

(2.10) and (2.11) imply that the conditional distribution of $\left(\left(Z_{1}, \alpha_{1}, \delta_{1}\right), \cdots,\left(Z_{n}, \alpha_{n}, \delta_{n}\right)\right)$ under $X_{(n)}=x_{(n)}$ is

$$
\prod_{i=1}^{n}\left\{\left[p \bar{G}_{i}\left(z_{i}\right) f\left(z_{i} ; x_{i}^{T} \beta\right)\right]^{\bar{\alpha}_{i} \bar{\delta}_{i}}\left[(1-p) F\left(z_{i} ; x_{i}^{T} \beta\right) g_{i}\left(z_{i}\right)\right]^{\bar{\alpha}_{i}\left(1-\bar{\delta}_{i}\right)} \cdot\left[\bar{F}\left(z_{i} ; x_{i}^{T} \beta\right) g_{i}\left(z_{i}\right)\right]^{1-\bar{\alpha}_{i}} \mu\left(d z_{i}\right)\right\}, n \geq 1
$$

The conditional probability measure corresponding to (2.13) is written as $P_{\beta}\left(\cdot \mid X_{\infty}=x_{\infty}\right)$. Meanwhile, let $E_{\beta}^{x_{\infty}}(\cdot)$ and $\operatorname{Var}_{\beta}^{x_{\infty}}(\cdot)$ denote the conditional expectation and conditional variance under the conditional probability measure $P_{\beta}\left(\cdot \mid X_{\infty}=x_{\infty}\right)$, respectively. Set $\beta_{0}$ do- note the real value of $\beta$. For notational simplicity, let $E^{x_{\infty}}(\cdot) \equiv E_{\beta}^{x_{\infty}}(\cdot)$ and $\operatorname{Var}^{x_{\infty}}(\cdot) \equiv \operatorname{Var}_{\beta}^{x_{\infty}}(\cdot)$. (2.13) implies that the joint distribution of is $\left(\left(Z_{1}, \alpha_{1}, \delta_{1}, X_{1}\right), \cdots,\left(Z_{n}, \alpha_{n}, \delta_{n}, X_{n}\right)\right)$

$$
\prod_{i=1}^{n}\left\{\left[p \bar{G}_{i}\left(z_{i}\right) f\left(z_{i} ; x_{i}^{T} \beta\right)\right]^{\bar{\alpha}_{i} \bar{s}_{i}}\left[(1-p) F\left(z_{i} ; x_{i}^{T} \beta\right) g_{i}\left(z_{i}\right)\right]^{\bar{\alpha}_{i}(1-\bar{\delta})_{i}} \cdot\left[\bar{F}\left(z_{i} ; x_{i}^{T} \beta\right) g_{i}\left(z_{i}\right)\right]^{1-\bar{\alpha}_{i}} \mu\left(d z_{i}\right) \tau_{i}\left(x_{i}\right) \mu\left(d x_{i}\right)\right\}
$$

The probability measure (unconditional) corresponding to (2.14) is denoted as $P_{\beta}(\cdot)$. Meanwhile, let $E_{\beta}(\cdot)$ and $\operatorname{Var}_{\beta}(\cdot)$ denote the expectation and variance under the probability measure $P_{\beta}(\bullet)$, respectively. For notational simplicity, let

$$
P(\cdot)=P_{\beta}(\cdot), E(\cdot)=E_{\beta}(\cdot) \text { and } \operatorname{Var}(\cdot)=\operatorname{Var}_{\beta}(\cdot)
$$

It is that the parameters in (2.14) are studied by us.

\section{Main Results}

Furthermore, from (2.14) we get the likelihood function of

as follows

$$
\left(\left(Z_{1}, \alpha_{1}, \delta_{1}, X_{1}\right), \cdots,\left(Z_{n}, \alpha_{n}, \delta_{n}, X_{n}\right)\right)
$$

$$
L\left(\beta, Z_{1}, \alpha_{1}, \delta_{1}, X_{1}, \cdots, Z_{n}, \alpha_{n}, \delta_{n}, X_{n}\right)
$$

$$
\begin{aligned}
\equiv & \prod_{i=1}^{n}\left\{\left[p \bar{G}_{i}\left(Z_{i}\right) f\left(Z_{i} ; X_{i}^{T} \beta\right)\right]^{\alpha_{i} \delta_{i}}\right. \\
& \times\left[(1-p) F\left(Z_{i} ; X_{i}^{T} \beta\right) \cdot g_{i}\left(Z_{i}\right)\right]^{\alpha_{i}\left(1-\delta_{i}\right)} \\
& \left.\times\left[\bar{F}\left(Z_{i} ; X_{i}^{T} \beta\right) g_{i}\left(Z_{i}\right)\right]^{1-\alpha_{i}} \tau_{i}\left(X_{i}\right)\right\}, n \geq 1
\end{aligned}
$$

Taking the logarithm to (3.1) and dropping the terms which are free of $\beta$ yield the logarithm likelihood function:

$$
\begin{aligned}
l_{n}^{*}(\beta)= & \sum_{i=1}^{n}\left[\alpha_{i} \delta_{i} \log f\left(Z_{i} ; X_{i}^{T} \beta\right)+\alpha_{i}\left(1-\delta_{i}\right) \cdot\right. \\
& \left.\log F\left(Z_{i} ; X_{i}^{T} \beta\right)+\left(1-\alpha_{i}\right) \log \bar{F}\left(Z_{i} ; X_{i}^{T} \beta\right)\right] \\
= & l_{n}\left(\beta ; Z_{1}, \alpha_{1}, \delta_{1}, X_{1}, \cdots, Z_{n}, \alpha_{n}, \delta_{n}, X_{n}\right),
\end{aligned}
$$

where $l_{n}\left(\beta ; Z_{1}, \alpha_{1}, \delta_{1}, x_{1}, \cdots, Z_{n}, \alpha_{n}, \delta_{n}, x_{n}\right)$ is the logarithm likelihood function defined in Xiao and Liu [8]. 
We have the score function

$$
\begin{aligned}
T_{n}^{*}(\beta) & \equiv \partial l_{n}^{*}(\beta) / \partial \beta=\sum_{i=1}^{n} X_{i}\left[\alpha_{i} \delta_{i} Z_{i}-\dot{b}\left(X_{i}^{T} \beta\right)+\frac{\alpha_{i}\left(1-\delta_{i}\right)}{F_{i}\left(Z_{i} ; X_{i}^{T} \beta\right)} \int_{-\infty}^{Z_{i}} y f_{i}\left(y ; X_{i}^{T} \beta\right) \mu(d y)\right. \\
& \left.+\frac{\left(1-\alpha_{i}\right)}{\bar{F}\left(Z_{i} ; X_{i}^{T} \beta\right)} \int_{Z_{i}}^{\infty} y f_{i}\left(y ; X_{i}^{T} \beta\right) \mu(d y)\right] \equiv T_{n}\left(\beta ; Z_{1}, \alpha_{1}, \delta_{1}, X_{1}, \cdots, Z_{n}, \alpha_{n}, \delta_{n}, X_{n}\right),
\end{aligned}
$$

where $T_{n}\left(\beta ; Z_{1}, \alpha_{1}, \delta_{1}, X_{1}, \cdots, Z_{n}, \alpha_{n}, \delta_{n}, X_{n}\right)$ is defined as in Xiao and Liu [8]. And

$$
\begin{aligned}
H_{(n)}^{*}(\beta) \equiv & -\frac{\partial^{2} l_{n}^{*}(\beta)}{\partial \beta \partial \beta^{T}}=\sum_{i=1}^{n} X_{i} X_{i}^{T}\left\{\ddot{b}\left(X_{i}^{T} \beta\right)+\alpha_{i}\left(1-\delta_{i}\right)\left[\frac{1}{F_{i}^{2}\left(Z_{i} ; X_{i}^{T} \beta\right)}\left(\int_{-\infty}^{Z_{i}} y f_{i}\left(y ; X_{i}^{T} \beta\right) \mu(d y)\right)^{2}\right.\right. \\
& \left.-\frac{1}{F_{i}\left(Z_{i} ; X_{i}^{T} \beta\right)} \int_{-\infty}^{Z_{i}} y^{2} f_{i}\left(y ; X_{i}^{T} \beta\right) \mu(d y)\right]+\left(1-\alpha_{i}\right)\left[\frac{1}{\overline{F_{i}^{2}\left(Z_{i} ; X_{i}^{T} \beta\right)}}\left(\int_{Z_{i}}^{\infty} y f_{i}\left(y ; X_{i}^{T} \beta\right) \mu(d y)\right)^{2}\right. \\
& \left.\left.-\frac{1}{\bar{F}_{i}\left(Z_{i} ; X_{i}^{T} \beta\right)} \int_{Z_{i}}^{\infty} y^{2} f_{i}\left(y ; X_{i}^{T} \beta\right) \mu(d y)\right]\right\} \\
= & H_{n}\left(\beta ; Z_{1}, \alpha_{1}, \delta_{1}, x_{1}, \cdots, Z_{n}, \alpha_{n}, \delta_{n}, x_{n}\right)
\end{aligned}
$$

where $H_{n}\left(\beta ; Z_{1}, \alpha_{1}, \delta_{1}, x_{1}, \cdots, Z_{n}, \alpha_{n}, \delta_{n}, x_{n}\right)$ is defined as in Xiao and Liu [8]. Write

$$
\begin{aligned}
\Lambda_{n}^{*}(\beta) & \equiv \Lambda_{n}^{*}\left(\beta ; x_{1}, \cdots, x_{n}\right) \equiv E_{\beta}^{x_{\infty}}\left[T_{n}^{*}(\beta)\left(T_{n}^{*}(\beta)\right)^{T}\right] \\
& =-E_{\beta}^{x_{\infty}} H_{n}^{*}(\beta)=\Lambda_{n}\left(\beta ; x_{1}, \cdots, x_{n}\right),
\end{aligned}
$$

where $\Lambda_{n}\left(\beta ; x_{1}, \cdots, x_{n}\right)$ is defined as in Xiao and Liu [8].

The solution of the logarithm likelihood equation

$$
T_{n}^{*}(\beta)=0
$$

is written as

$$
\tilde{\beta}_{n} \equiv \tilde{\beta}_{n}\left(Z_{1}, \alpha_{1}, \delta_{1}, X_{1}, \cdots, Z_{n}, \alpha_{n}, \delta_{n}, X_{n}\right) .
$$

(3.3) and (3.4) imply that

$$
\tilde{\beta}_{n} \equiv \hat{\beta}_{n}\left(Z_{1}, \alpha_{1}, \delta_{1}, X_{1}, \cdots, Z_{n}, \alpha_{n}, \delta_{n}, X_{n}\right),
$$

where $\hat{\beta}_{n}\left(Z_{1}, \alpha_{1}, \delta_{1}, X_{1}, \cdots, Z_{n}, \alpha_{n}, \delta_{n}, X_{n}\right)$ is defined as in Xiao and Liu [8]. The norm of matrix $A=\left(a_{i j}\right)_{p \times q}$ is defined as $\|A\|=\sqrt{\sum_{i=1}^{p} \Sigma_{j=1}^{q} a_{i j}^{2}}$. We write $<\cdot, \cdot>$ as the usual inner product and $e_{s}$ as the sth canonical basis in $\mathbb{R}^{q}$. Let

$$
\begin{gathered}
\Upsilon_{1}(z, \theta)=F^{-1}(z, \theta) \int_{-\infty}^{z} y f(y ; \theta) \mu(d y) \\
\Upsilon_{2}(z, \theta)=F^{-1}(z, \theta) \int_{-\infty}^{z} y^{2} f(y ; \theta) \mu(d y) \\
\Upsilon_{3}(z, \theta)=\bar{F}^{-1}(z, \theta) \int_{z}^{\infty} y f(y ; \theta) \mu(d y) \\
\Upsilon_{4}(z, \theta)=\bar{F}^{-1}(z, \theta) \int_{z}^{\infty} y^{2} f(y ; \theta) \mu(d y)
\end{gathered}
$$

and $\Lambda_{n}(\beta)=E_{\beta}\left[T_{n}(\beta) T_{n}^{T}(\beta) \mid X_{(n)}=x_{(n)}\right]$.

We state the following assumptions:

( $\bar{C} 1$ ) For all $i \geq 1$, for all $\beta \in B, X_{i}^{T} \beta \in \Theta_{0}$, a.s., where $X_{i} \in \mathfrak{\aleph}$. Here $\mathfrak{\aleph}$ is compact.

( $\bar{C} 2) Q\left(\beta_{0} ; X_{\infty}\right) \equiv \lim _{n \rightarrow \infty}\left(n \Lambda_{n}^{-1}\left(\beta_{0} ; X_{(n)}\right)\right)$ is a q-order positive define matrix.

( $\bar{C} 3$ ) For all $\beta_{1}, \beta_{2} \in B$,

$$
\begin{aligned}
& \left|\Upsilon_{1}^{2}\left(z ; x^{T} \beta_{1}\right)-\Upsilon_{1}^{2}\left(z ; x^{T} \beta_{2}\right)\right| \leq L_{1}(z, x)\left|\beta_{1}-\beta_{2}\right|, \\
& \left|\Upsilon_{2}\left(z ; x^{T} \beta_{1}\right)-\Upsilon_{2}\left(z ; x^{T} \beta_{2}\right)\right| \leq L_{2}(z, x)\left|\beta_{1}-\beta_{2}\right|, \\
& \left|\Upsilon_{3}^{2}\left(z ; x^{T} \beta_{1}\right)-\Upsilon_{3}^{2}\left(z ; x^{T} \beta_{2}\right)\right| \leq L_{3}(z, x)\left|\beta_{1}-\beta_{2}\right|, \\
& \left|\Upsilon_{4}\left(z ; x^{T} \beta_{1}\right)-\Upsilon_{4}\left(z ; x^{T} \beta_{2}\right)\right| \leq L_{4}(z, x)\left|\beta_{1}-\beta_{2}\right|,
\end{aligned}
$$

where

$$
\sup _{i \geq 1} E\left[L_{j}^{b}\left(Z_{i} ; x^{T}\right) \mid X_{\infty}\right] \leq L_{j}<\infty, a . s . j=1,2,3,4, b>1 .
$$

$$
\text { ( } \bar{C} 4) \quad \forall \beta \in B, i \geq 1, E_{\beta}^{X_{\infty}}\left[t^{2}\left(X_{i}^{T} \beta\right)\right]>0 \text {, a.s. }
$$

It is also easy to see that the conditions in the present paper imply the conditions (C1), (C2), (C3) and (C4) given in Xiao and Liu[8]. So, there almost sure exists the maximum likelihood estimator of $\beta_{0}$. Hence, our first result states a law of the iterated logarithm for the maximum likelihood estimator of $\beta_{0}$.

Theorem 3.1. Under conditions $(\bar{C} 1),(\bar{C} 2),(\bar{C} 3)$ and $(\bar{C} 4)$, if $\hat{\beta}_{n}$ is the MLE of $\beta_{0}$, then for $1 \leq s \leq q$, we have 


$$
P_{\beta_{0}}\left\{\limsup _{n \rightarrow \infty} \sqrt{\frac{n}{2 \log \log n}}<e_{s}, \hat{\beta}_{n}-\beta_{0}>=\sqrt{e_{s}^{T} Q\left(\beta_{0}\right) e_{s}} \mid X_{\infty}\right\}=1 \text {, a.s. }
$$

and

$$
P_{\beta_{0}}\left\{-\liminf _{n \rightarrow \infty} \sqrt{\frac{n}{2 \log \log n}}<e_{s}, \hat{\beta}_{n}-\beta_{0}>=-\sqrt{e_{s}^{T} Q\left(\beta_{0}\right) e_{s}} \mid X_{\infty}\right\}=1 \text {, a.s. }
$$

Proof. For arbitrarily given $x_{\infty}=\left(x_{1}, \cdots, x_{n}, \cdots\right)$, we regard the conditional probability measure $P\left(\cdot \mid X_{\infty}=x_{\infty}\right)$ as the probability measure $P(\cdot)$ defined in Xiao and Liu [8], and note that as $X_{\infty}=x_{\infty}$ is given, MLE $\tilde{\beta}_{n}$ is equivalent to MLE

$\hat{\beta}_{n}=\hat{\beta}_{n}\left(Z_{1}, \alpha_{1}, \delta_{1}, x_{1}, \cdots, Z_{n}, \alpha_{n}, \delta_{n}, x_{n}\right)$ obtained in Xiao and Liu [6]. Thus, Remark 2.1 implies Theorem 2.1 in Xiao and Liu [8], and hence we have the desired results.

Remark 3.1. Under the conditions of Theorem 3.1, we take expectations for the results above and immediately get

and

$$
P_{\beta_{0}}\left\{\limsup _{n \rightarrow \infty} \sqrt{\frac{n}{2 \log \log n}}<e_{s}, \hat{\beta}_{n}-\beta_{0}>=-\sqrt{e_{s}^{T} Q\left(\beta_{0}\right) e_{s}}\right\}=1,
$$

$$
P_{\beta_{0}}\left\{-\liminf _{n \rightarrow \infty} \sqrt{\frac{n}{2 \log \log n}}<e_{s}, \hat{\beta}_{n}-\beta_{0}>=-\sqrt{e_{s}^{T} Q\left(\beta_{0}\right) e_{s}}\right\}=1
$$

Note that Theorem 3.1 establishes a law of iterated logarithm for each component of $\hat{\beta}_{n}$. Our next result is a Chung type law of iterated logarithm. To this aim, we add and additional condition. For notational simplicity, let $\omega_{i}(s)=e_{s}^{T} Q\left(\beta_{0}\right) X_{i} t\left(X_{i}^{T} \beta_{0}\right)$.

Then

$$
\begin{aligned}
& \qquad \omega_{i}^{2}(s)=e_{s}^{T} Q\left(\beta_{0}\right) X_{i} X_{i}^{T} t^{2}\left(X_{i}^{T} \beta_{0}\right) Q^{T}\left(\beta_{0}\right) e_{s} \\
& \qquad P_{\beta_{0}}\left\{\operatorname { l i m i n f } _ { n \rightarrow \infty } \sqrt { \frac { \operatorname { l o g } \operatorname { l o g } n } { n } } \operatorname { m a x } _ { 1 \leq i \leq n } \left\{i \mid<e_{s}, \hat{\beta}_{n}\right.\right. \\
& \text { Proof. In the way similar to that of Theorem 3.1, we }
\end{aligned}
$$$$
P_{\beta_{0}}\left\{\liminf _{n \rightarrow \infty} \sqrt{\frac{\log \log n}{n}} \max _{1 \leq i \leq n}\left\{i\left|<e_{s}, \hat{\beta}_{n}-\beta_{0}>\right|\right\}=\frac{\pi}{\sqrt{8}} \sqrt{e_{s}^{T} Q\left(\beta_{0}\right) e_{s}} \mid X_{\infty}\right\}=1 \text {, a.s. }
$$

We make the following assumption:

$$
\begin{array}{r}
(\bar{C} 5) \inf _{k \in I} E\left[\omega_{k}^{2}(s) \mid X_{\infty}\right]>0, \text { a.s., where } \\
I=\left\{i: E\left[\omega_{i}^{2}(s) \mid X_{\infty}\right]>0\right\} \text { a.s.. }
\end{array}
$$

Theorem 3.2. Under conditions $(\bar{C} 1),(\bar{C} 2),(\bar{C} 3)$, $(\bar{C} 4)$ and $(\bar{C} 5)$, if $\hat{\beta}_{n}$ is the MLE of $\beta_{0}$, then for $1 \leq s \leq q$, we have immediately obtain the desired result. we take expectations for the results above and immediately get
Remark 3.2. Under the conditions of Theorem 3.2,

$$
P_{\beta_{0}}\left\{\liminf _{n \rightarrow \infty} \sqrt{\frac{\log \log n}{n}} \max _{1 \leq i \leq n}\left\{i\left|<e_{s}, \hat{\beta}_{n}-\beta_{0}>\right|\right\}=\frac{\pi}{\sqrt{8}} \sqrt{e_{s}^{T} Q\left(\beta_{0}\right) e_{s}}\right\}=1
$$

\section{Conclusions}

The results obtained in the present paper are based on the case that the link function is a natural link function. However, Ding and Chen [9] gave the consistency and asymptotic normality of MLE $\hat{\beta}_{n}$ of GLM under the case that the link function is of non-natural link, hence, the academicians who are interested in GLM may furthermore investigate the iterated logarithm law and Chung type iterated logarithm law of MLE $\hat{\beta}_{n}$ of GLM under the case that the link function is of non-natural link.

\section{Acknowledgements}

The authors would like to thanks the unknown referees for helpful comments.

\section{References}

[1] J. A. Nelder and R. W. Wedderburn, "Generalized Linear Models,” Journal of the Royal Statistical Society: Series 
A, 135, Part 3, 1972, pp. 370-384. doi:10.2307/2344614

[2] L. Fahrmeir and H. Kaufmann, "Consistency and Asymtotic Normality of the Maximum Likelihood Estimator in Generalized Linear Models,” Annals of Statistics, Vol. 13, No. 1, 1985, pp. 342-368. doi:10.1214/aos/1176346597

[3] T. Elperin and I. Gertsbak, "Estimation in a Random Censoring Model with Incomplete Information: Exponential Lifetime Distribution," IEEE Transactions on Reliability, Vol. 37, No. 2, 1988, pp. 223-229. doi:10.1109/24.3745

[4] T. L. Lai and C. Z. Wei, "Least Squares Estimates in Stochastic Regression Models with Applications to Identification and Control of Dynamic Systems," Annals of Statistics, Vol. 10, No. 1, 1982, pp. 154-186. doi:10.1214/aos/1176345697

[5] S. L. Zeger and M. R. Karim "Generalized Linear Models with Random Effects; A Gibbs' Sampling Approach," Journal of the American Statistical Association, Vol. 86, No. 413, 1991, pp. 79-86. doi:10.2307/2289717
[6] Z. H. Xiao and L. Q. Liu, "MLE of Generalized Linear Model Randomly Censored with Incomplete Information,” Acta Mathematica Scientia, Vol. 3(A), 2008, pp. 553-564.

[7] Z. H. Xiao and L. Q. Liu. "Laws of Iterated Logarithm for Quasi-Maximum Likelihood Estimator in Generalized Linear Model," Journal of statistical planning and inference, Vol. 138, No. 3, 2008, pp. 611-617. doi:10.1016/j.jspi.2006.12.006

[8] Z. H. Xiao and L. Q. Liu, "Laws of Iterated Logarithm for MLE of Generalized Linear Model Randomly Censored with Incomplete Information," Statistics and Probability Letters, Vol. 79, No. 6, 2009, pp. 789-796. doi:10.1016/j.spl.2008.11.016

[9] J. L. Ding and X. R. Chen, “Asymptotic Properties of the Maximum Likelihood Estimate in Generalized Linear Models with Stochastic Regressors," Acta Mathematica Sinica, Vol. 22, No. 6, 2006, pp. 1679-1686. doi:10.1007/s10114-005-0693-3 